\title{
Planets orbiting stars more massive than the Sun
}

\author{
E. W. Guenther ${ }^{1}$, D. Sebastian ${ }^{1}$, D. Gandolfi ${ }^{2}$, P. Montañés-Rodríguez ${ }^{3,4}$, St. Geier ${ }^{5,6}$, Sz. Csizmadia $^{7}$, \\ F. Cusano ${ }^{8}$, H. J. Deeg ${ }^{3,4}$, M. Fridlund ${ }^{9}$, S. Grziwas ${ }^{10}$, U. Heber ${ }^{6}$, C. Heuser ${ }^{6}$, F. Rodler ${ }^{11,12,13}$, and L. Tal-Or ${ }^{14}$, \\ and the CoRoT-team*
}

Thüringer Landessternwarte Tautenburg, 07778 Tautenburg, Germany e-mail: guenther@tls-tautenburg.de

2 Zentrum für Astronomie Heidelberg,, Landessternwarte Heidelberg-Königstuhl 12, D-69117 Heidelberg, Germany

3 Instituto de Astrofísica de Canarias, 38205 La Laguna, Tenerife, Spain

4 Dpto. de Astrofísica, Universidad de La Laguna, 38206 La Laguna, Tenerife, Spain

5 ESO, Karl-Schwarzschild-Str. 285748 Garching bei München, Germany

6 Astronomisches Institut, der Universität Erlangen-Nürnberg, Dr. Remeis-Sternwarte, Sternwartstr. 7, 96049 Bamberg, Germany

7 Institute of Planetary Research, German Aerospace Center, Rutherfordstraße 2, 12489 Berlin, Germany

8 INAF-Osservatorio Astronomico di Bologna, Via Ranzani 1, 1-40127 Bologna, Italy

9 Max-Planck-Institut für Astronomie, Königstuhl 17, D-69117 Heidelberg, Germany

10 Rheinisches Institut für Umweltforschung an der Universiät zu Köln, Aachener Straße 209, 50931 Köln, Germany

11 Harvard-Smithsonian Center for Astrophysics, Cambridge, MA 02138, USA

12 Institut de Cienci'e s de l'Espai (CSIC-IEEC), Campus UAB, Fac. Ciencies, C5 p2, E-08193 Barcelona, Spain

13 Max-Planck-Institut für Astronomie, Königstuhl 17, D-691 17 Heidelberg, Germany

14 Institut fur Astrophysik (IAG), Friedrich-Hund-Platz 1, 37077 Göttingen, Germany

\section{Introduction}

Although thousands of transiting extrasolar planets have been discovered, less than $4 \%$ orbit stars in the mass-range $\mathrm{M}_{\text {star }}=1.3-2.1 \mathrm{M}_{\odot}$. This means that we know only very little about planets of these intermediate mass stars (IMSs). This is unfortunate, because studies with the SPITZER telescope demonstrate that the life-time of planet-forming disks is on average about half as long as that for solarlike stars $\left(\tau_{\text {disk }}=1.2\right.$ Myrs for $M_{*} \geq 1.3 M_{\odot}$ compared to $\tau_{\text {disk }}=2.5$ Myrs for $M_{*} \sim 1 M_{\odot}$; Mamajek 2009). Thus, although the disks of IMSs are on average more massive than those of solar-like stars, there is, on average, less time to form planets. However most theories still predict a higher

\footnotetext{
* Based on observations obtained with CoRoT. The CoRoT space mission, launched on December 27, 2006, has been developed and is operated by the CNES, with the contribution of Austria, Belgium, Brazil, ESA (RSSD and Science Program), Germany and Spain. The ground-based observations were obtained at the European Southern Observatory, with the the Large Binocular Telescope, with the 2.2-m and 3.5-m telescopes at CAHA, with the NOT, with the 2.1-m telescope at McDonald observatory, with the Alfred-Jensch telescope, and with the IAC-80-cm telescope.
}

frequency of massive planets of IMSs than for solar-like stars (Laughlin et al. 2004; Ida \& Lin 2005; Kennedy \& Kenyon 2008; Alibert et al. 2011; Hasegawa \& Pudritz 2013), but not all agree (Kornet et al. 2004; Boss 2004).

Studies of giant stars and direct imaging surveys confirm that the frequency of massive planets is higher for IMSs than for solar-like stars (Vigan et al. 2012; Johnson et al. 2010a, 2010b). This means that massive planets can form within about 1.2 Myrs. However, these types of surveys are limited to planets at orbital distances larger than $0.5 \mathrm{AU}$.

We thus do not know the frequency of close-in planets of IMSs, as only very few discoveries have been made (e.g. Collier Cameron et al. 2010). The question is whether they can not only form but also migrate inwards while the disk is still there. Gas-giant planets can migrate via the socalled Type II migration in by planet-disk interaction (e.g., Lin et al. 1996). If Type II migration were the dominant process, the frequency of close-in planets would strongly depend on the migration-rate (Williams \& Cieza 2011). By measuring the frequency of close-in, massive planets, we thus constrain speed of Type II migration. Based on data obtained for subgiants stars, Bowler et al. (2010) argue that there is a significant lack of close-in planets of IMSs. 
In their sample of 31 stars, they can rule out planets of more than $1.0 \mathrm{M}_{\text {Jup }}$ within $1 \mathrm{AU}$. They furthermore argue that this lack of planets is not due to an observational bias but must be real.

Planets of stars with $\mathrm{M}_{\text {star }} \geq 1.2 \mathrm{M}_{\odot}$ (corresponding to $T_{\text {eff }} \geq 6250 \mathrm{~K}$ ) are, in contrast to planets of lower-mass stars, quite often misaligned (Winn et al. 2010). This result is surprising, since Beaugé et al. (2012) has shown that the interaction between the planet and the disk leads to a damping of the orbital inclination of the planets. Planets that migrate inwards via Type II migration should thus be co-planar. The discovery that planets of IMSs are often misaligned thus led to intensive discussions (Albrecht et al. 2012; Albrecht et al. 2013; Chaplin et al. 2013; Hirano et al. 2012; Lai et al. 2011; Sanchis-Ojeda et al. 2012; Van Eylen 2014; Huber et al.2013; Xue et al. 2014).

Another interesting aspect is engulfment of close-in planets in IMSs during the post-main sequence phase. Which kind of planets are engulfed, and whether planets can survive the engulfment to reappear at later stages is still an open question. As shown in Villaver \& Livio (2009) tidal interactions lead to the engulfment of close-in planets when the star becomes a giant. The minimum distance at which a planet can survive depends on its mass (Kunitomo 2011). A planet with the mass of Jupiter orbiting a star with $1.5 \mathrm{M}_{\text {Sun }}$ has to be at a distance of more than $1.5 \mathrm{AU}$ to survive the red giant phase. The short-period planets that we discuss in this article are thus unlikely to survive the red giant phase.

The effects of the engulfment of brown dwarfs (BDs) and massive planets can have quite dramatic consequences for the star. The accretion of BDs and planets can induce the ejection of shells around giant stars, changes the abundance pattern, and lead to significant spin-up (Bear \& Soker 2012; Beuermann et al. 2012; Charpinet et al. 2011; Livio \& Soker 2002; Massarotti 2008; Pasquini et al. 2007; Politano et al. 2008; Siess \& Livio 1999a; Siess \& Livio 1999b; Silvotti et al. 2007).

The discovery of a post-red-giant host stars with giant planets orbiting as close as $0.116 \mathrm{AU}$, and two nearly Earth-sized bodies orbiting the hot B Subdwarf star (sdB) at distances of 0.0060 and 0.0076 AU shows that at least these planets must have survived the engulfment (Charpinet et al. 2011). According to Charpinet et al. (2011), the sdB-planets are the dense cores of evaporated giant planets that were transported closer to the star during the engulfment and triggered the mass loss necessary for the formation of the sdB. Furthermore, it has been suggested that close-in planets are also important for the shaping of planetary nebulae (Soker 1997; De Marco \& Soker 2011). However, this model requires that $20 \%$ of the planetary nebulae are affected by close-in planets. Thus, also in the context of stellar evolution it is important to find out, what the frequency of close-in, massive planets is.

In summary, the determination of the frequency of closein planets of IMSs sets important constraints for the theories of planet formation and evolution, and it is also important for our understanding of post-main sequence stellar evolution. We have therefore started a search for close-in transiting planets around IMSs using the CoRoT data. The main focus of our survey are planets of B-, and A-stars, because most planets of F-stars are expected to have been found already in the regular planet-survey of CoRoT.

\section{The survey}

Because of the lack of absorption lines, and the rapid rotation of B-, A-, and early F-stars, radial-velocity (RV) surveys for planets of these types of stars are not efficient (Desort 2009a; Desort 2009b; Guenther et al. 2009; Galland et al. 2006; Galland et al. 2010; Borgniet et al. 2014). Transit surveys are more suitable, because they do not suffer from these difficulties. The only challenges of transit surveys is that the planetary transit are shallower than for solar-like stars, and that many IMSs oscillate. Groundbased transit surveys have already discovered planets of IMSs, for example HD15082b (Collier Cameron et al. 2010). However, detecting planets of A-stars with ground-based telescopes is also not easy. Nevertheless, HD15082b is up to now the only known transiting planet of an A-star were the mass has been determined (Lehmann et al. 2014). The noise-level of CoRoT is $80 \mathrm{ppm}, 110 \mathrm{ppm}, 200 \mathrm{ppm}$ and $280 \mathrm{ppm}$ for a a single $3 \mathrm{~h}$ transit for stars of 11.5, 12.5, 13.5, and $14.5 \mathrm{mag}$, respectively (Auvergne et al. 2009). Since we are only interested in short-period planets with the size of Jupiter, the noise-levels are about 16, 22, 40, $56 \mathrm{ppm}$, if we limit our survey to the long runs (LRa-, LRc-, and the IRa-field $\left.{ }^{1}\right)$ and to planets with orbital periods $P \leq 6 d$, and if we co-add and phase-folded transits. A transit with a depth of $3400 \mathrm{ppm}$, as it is expected for a Jupiter-sized planet orbiting a A5V star $\left(R_{*}=1.7 \mathrm{R}_{\odot}\right)$ is thus easily detected by CoRoT. This means that CoRoT must have detected all transiting giant planets with short orbital periods in these fields. To determine the frequency of close-in planets and BDs, all we have to do is to find out whether the transits are false-positives (FPs), eclipsing binaries, or not. After excluding binaries, and all types of FPs, all remaining objects have to be planets or BDs. The main focus of our survey are B- and A-stars, because we expect that almost all planets of F-stars were already found in the main survey of CoRoT. CoRoT has in fact already discovered a number of planets and BDs orbiting stars more massive than the Sun (Table III.7.1).

According to EXODAT (Deleuil et al. 2009), CoRoT has observed 1126 B-stars, 18450 A-stars, 23826 F-stars, 40959 G-stars, $38601 \mathrm{~K}$-stars, and $2220 \mathrm{M}$-stars in the LRfields. Counting only stars classified as luminosity-class IV and V, there are 1044 B-stars, 18394 A-stars, 22578 F-stars, 29753 G-stars $6714 \mathrm{~K}$-stars, and 79 M-stars in these fields. Since CoRoT discovered 11 planets with $R_{\mathrm{p}} \geq 0.5 \mathrm{R}_{\mathrm{Jup}}$ with $P \leq 6 d$ of $\mathrm{G}, \mathrm{K}$, and M-stars in the LR-fields, we expect to find about 6 substellar-objects of A-, and B-stars, if the frequency of planets were the same. If there are as many close-in planets of IMSs as there are planets at larger distance, we should find about 8 of them.

\section{The candidates}

Given that, in the CoRoT survey, planet candidates are selected without any bias against certain spectral types of the host stars, we expect that many have alredy been identified in the main planet survey. However, since the discovery of planets transiting B- and A-type stars is not easy, it is very well possible that some planets of A- and

1 LRa stands for "Long-Run-galactic-Anti-centre field", LRc for "Long-Run-galactic-centre field", and IRa for "initial field". The IRa-field was also located in the "antic-centre" direction. 
Planets orbiting stars more massive than the Sun

Table III.7.1. Planets of stars more massive than $1.1 \mathrm{M}_{\odot}$ discovered by CoRoT.

\begin{tabular}{lcclcccl}
\hline \hline Name & $\begin{array}{c}\mathrm{M}_{\text {star }} \\
{\left[\mathrm{M}_{\odot}\right]}\end{array}$ & $\begin{array}{c}\text { Spec- } \\
\text { type }\end{array}$ & $\begin{array}{l}\text { Period } \\
{[\mathrm{d}]}\end{array}$ & $\begin{array}{c}\mathrm{R}_{\text {planet }} \\
{\left[\mathrm{R}_{\text {Jup }}\right]}\end{array}$ & $\begin{array}{c}\mathrm{M}_{\text {planet }} \\
{\left[\mathrm{M}_{\text {Jup }}\right]}\end{array}$ & $\begin{array}{c}\text { Density } \\
{\left[\mathrm{g} \mathrm{cm}^{-3}\right]}\end{array}$ & Ref. \\
\hline CoRoT-3b & $1.37 \pm 0.09$ & F3V & 4.3 & $1.01 \pm 0.07$ & $21.7 \pm 1.0$ & $26.4 \pm 5.6$ & Deleuil et al. 2008 \\
CoRoT-15b & $1.32 \pm 0.12$ & F7V & 3.0 & $1.12 \pm 0.23$ & $63.3 \pm 4.1$ & $59 \pm 35$ & Bouchy et al. 2011 \\
CoRoT-21b & $1.29 \pm 0.09$ & F8IV & 2.7 & $1.30 \pm 0.14$ & $2.53 \pm 0.37$ & $1.53 \pm 0.53$ & Pätzold et al. 2012 \\
CoRoT-11b & $1.27 \pm 0.05$ & F6V & 3.0 & $1.43 \pm 0.03$ & $2.33 \pm 0.34$ & $0.99 \pm 0.15$ & Gandolfi et al. 2010 \\
CoRoT-19b & $1.20 \pm 0.05$ & F9V & 3.9 & $1.29 \pm 0.03$ & $1.14 \pm 0.05$ & $0.51 \pm 0.05$ & Guenther et al. 2009 \\
CoRoT-4b & $1.16 \pm 0.02$ & F8V & $9.2^{1}$ & $1.17 \pm 0.05$ & $0.75 \pm 0.01$ & $0.58 \pm 0.15$ & Moutou et al. 2008 \\
CoRoT-22b & $1.15 \pm 0.08$ & G0IV & $9.7^{1}$ & $0.52 \pm 0.12$ & $<0.15$ & $<1.3$ & Moutou et al. 2014 \\
CoRoT-20b & $1.14 \pm 0.08$ & G2V & $9.2^{1}$ & $0.84 \pm 0.04$ & $4.24 \pm 0.23$ & $9.87 \pm 1.10$ & Deleuil et al. 2012 \\
CoRoT-23b & $1.14 \pm 0.08$ & G0V & 3.6 & $1.05 \pm 0.13$ & $2.8 \pm 0.3$ & $3.3 \pm 1.0$ & Rouan et al. 2012 \\
CoRoT-14b & $1.13 \pm 0.09$ & F9V & 1.5 & $1.09 \pm 0.07$ & $7.6 \pm 0.6$ & $7.3 \pm 1.5$ & Tingley et al. 2011 \\
CoRoT-34b & $\sim 1.4$ & F5V & 5.6 & $\sim 1.9$ & $<1.4$ & - & 2 \\
\hline
\end{tabular}

Notes. ${ }^{(1)} P \geq 6$ d, thus object should not be counted. ${ }^{(2)}$ Planet candidate that is being studied, the CoRoT number is preliminary.

Table III.7.2. New candidates for substellar objects.

\begin{tabular}{llcclcl}
\hline \hline Win-ID & $\begin{array}{l}\text { Spec. } \\
\text { type }\end{array}$ & $\begin{array}{c}\mathrm{V} \\
{[\mathrm{mag}]}\end{array}$ & $\begin{array}{c}\mathrm{M}_{\text {star }} \\
{\left[\mathrm{M}_{\odot}\right]}\end{array}$ & $\begin{array}{l}\text { Period } \\
{[\mathrm{d}]}\end{array}$ & $\begin{array}{c}\text { Depth } \\
{[\%]}\end{array}$ & Remarks \\
\hline LRa02_E1_1475 $^{1}$ & A4V & 14.2 & 2.3 & 2.12 & 0.3 & BD or low-mass stellar companion \\
LRc07_E2_0307 $^{2}$ & F3V & 13.1 & 1.5 & 5.15 & 0.2 & planet \\
LRc09_E2_3322 $^{1}$ & A5IV & 15.7 & 2.1 & 3.2 & 1.04 & planet candidate \\
\hline IRa01_E2_2721 $^{2}$ & A6V & 13.0 & 2.0 & 0.61 & 0.8 & UVES: $\mathrm{M}_{\mathrm{p}} \leq 10 \mathrm{M}_{\text {Jup }}$ \\
LRa01_E2_0963 $^{2}$ & F0V & 13.8 & 1.7 & 4.65 & 0.3 & FIES+CAFÉ: $\mathrm{M}_{\mathrm{p}} \leq 30 \mathrm{M}_{\text {Jup }}$ \\
LRa02_E2_4150 $^{1}$ & B5V & 11.0 & 6.0 & $8.17^{3}$ & 0.7 & FIES: $\mathrm{M}_{\mathrm{p}} \leq 90 \mathrm{M}_{\text {Jup }}$ \\
LRc03_E2_2657 $^{1}$ & A7V & $13.1^{4}$ & 1.9 & 5.15 & 0.2 & UVES: $\mathrm{M}_{\mathrm{p}} \leq 15 \mathrm{M}_{\text {Jup }}$ \\
LRc08_E2_4203 $^{1}$ & A5V & 15.6 & 2.1 & 3.28 & 0.35 & UVES: $\mathrm{M}_{\mathrm{p}} \leq 15 \mathrm{M}_{\text {Jup }}$ \\
LRc10_E2_3265 $^{1}$ & A5V & 14.5 & 2.1 & 4.83 & 0.08 & TWIN: $\mathrm{M}_{\mathrm{p}} \leq 30 \mathrm{M}_{\text {Jup }}$ \\
\hline IRa01_E1_4591 $^{1}$ & A7V & 13.9 & 1.9 & 4.29 & 0.3 & SB1, or SB2 \\
LRa01_E2_0203 & F5IV & 11.6 & 1.3 & 1.86 & $<1.0$ & UVES: SB2 \\
LRa01_E2_1578 & F5IV & 13.4 & 1.3 & $16.06^{3}$ & 0.6 & SB1 \\
LRa02_E1_0725 & A5IV & 13.1 & 2.1 & $9.09^{3}$ & 1.4 & SB1 \\
LRa02_E2_1023 & F4V & 13.4 & 1.4 & 0.78 & 0.2 & LC-analysis: binary \\
LRc02_E1_0132 & F6II & 11.9 & 1.3 & 3.69 & & SB2 \\
LRc07_E2_0108 & A9IV & 11.2 & 1.8 & $14.45^{3}$ & 1.8 & SB1 \\
LRc07_E2_0146 & F8V & 12.5 & 1.2 & 0.49 & & LC-analysis: binary \\
LRc07_E2_0187 & F8V & 12.8 & 1.2 & 3.88 & 0.09 & LC-analysis: binary \\
LRc07_E2_0482 & F7V & 12.9 & 1.2 & 1.82 & & SB2 \\
\hline
\end{tabular}

Notes. ${ }^{(1)}$ Object found in regular CoRoT survey. ${ }^{(2)}$ Object found in this survey. ${ }^{(3)}$ Period $P \geq 6$, thus not strictly part of the survey.

B-stars might have been overlooked. We thus used the information given in EXODAT (Deleuil et al. 2009) to select all A-, and B-stars, as well as early F-stars observed in the IRa, LRa and LRc fields and analysed their lightcurves in detail. Altough we limit our survey to $P \leq 6 d$, we hesitated to throw away candidates with longer periods. From this list of stars we selected all potentially interesting candidates that had transits less than $1.5 \%$ deep. We also collected all so called "No-CCF-stars". These are targets with planet-like transit signals that have been already observed at least once by the spectroscopic follow-up program of CoRoT. Cross-correlation function (CCF) with a G-type template/mask show no significant correlation peak, implying that these stars are either rapid rotators, or earlytype objects, or both. We reanalyzed the acquired spectra and found that most of these objects are indeed rapidly rotating early-type stars. Table III.7.2 gives an overview of candidates identified.

The detailed reanalysis of the light-curves of LRa02. E2_1023, LRc07_E2_0146, LRc07_E2_0187 showed that these stars are binaries with very shallow secondary eclipses. For the remaining candidates, we obtained 
RV-measurements with the Echelle spectrographs SANDIFORD (McDonald 2.1-m telescope), CAFÉ (Calar Alto 2.2-m telescope), and FIES (NOT 2.6-m telescope) and the medium resolution spectrograph TWIN (Calar Alto 3.5-m telescope). These observations showed that another seven candidates are spectroscopic binaries. However, the fact that we are able to detect binaries with such shallow eclipses shows that CoRoT has the potential to detect giant planets around B- and A-stars. Although our primary goal was to find planets of B- and A-stars, we also included early F-stars in order not to miss any planet of an A-star just because of a slight mistyping of the spectral type.

As a next step, we obtained observing time with UVES on the VLT for the best candidates. Although the UVES spectra are of superb quality, we could only obtain upper limits for the companion masses of IRa01_E2_2721, LRc03_E2_2657, and LRc08_E2_4203. It is thus still possible that these are planets but it will not be easy to show that. Other good candidates are LRa02_E1_1475, LRc09_E2_3322, LRa02_E2_4150, and LRc07_E2_0307. It is interesting to note that the most promissing candidates were already found in the regular planet survey of CoRoT. This shows that the regular planet survey is essentially complete for giant, close-in planets. In this article, we will mostly discuss LRc07_E2_0307 and LRa02_E2_4150, because LRa02_E1_1475 and LRc09_E2_3322 will be described in forthcoming articles.

\section{The candidate LRc07_E2_0307}

LRc07_E2_0307 was continuously observed with the CoRoT satellite from 2011 April 8 (HJD: 2455660.094879911) to June 28 (HJD: 2455741.311633067) over a period of 81.2 days in the chromatic mode. The coordinates, proper motion, and brightnesses of the stars are given in Table III.7.3. In total 218,441 data-points were obtained in three colours with a 32 -second time sampling. Fifteen transits can already be seen in the raw-data. A low-resolution spectrum obtained with the NASMYTH spectrograph of the Alfred-Jensch telescope in Tautenburg shows that it is an F3V or F5IV star, which is in agreement with the temperature and $\log (\mathrm{g})$-values derived from the high-resolution spectra (Table III.7.3).

In order to prove that this star has a substellar companion, we have shown that it is not an FP, and that the companion is not a low-mass star. As shown by Almenara et al. (2009) most of the binaries and FPs can already be identified with a very detailed analysis of the light-curve. After this had been done, we obtained seeing-limited images in- and out-of transit to exclude that it is an FP (see Deeg et al. 2009 for details on this method). We obtained two sets of data, one with the IAC-80-cm telescope, the other with the WISE-1-m-telescope. Both the observations show that none of the stars within CoRoT photometric mask with a distance larger than $2^{\prime \prime}$ is the source of the transit signal. The transit is even cleraly seen in the data obtained with the WISE-1-m-telescope, which also helps to improve the ephemeris.

However, seeing limited observation can only confirm that the transiting object is within $2^{\prime \prime}$ from the target, not that the transiting object is exactly this star. The next step thus had to be AO-observations, which we obtained
Table III.7.3. Parameters of the planet and the star LRc07_E2_0307.

\begin{tabular}{ll}
\hline \hline Coordinates & \\
\hline RA (2000) & $18^{h} 31^{m} 00.241^{1}$ \\
Dec (2000) & $+07^{\circ} 11^{\prime} 00^{\prime} \cdot 6^{1}$ \\
Brigthness & \\
\hline B & $13.724 \pm 0.045^{2}$ \\
V & $13.055 \pm 0.066^{2}$ \\
R & $12.742 \pm 0.063^{2}$ \\
I & $12.436 \pm 0.02^{2}$ \\
J & $11.859 \pm 0.026^{3}$ \\
H & $11.641 \pm 0.022^{3}$ \\
K & $11.592 \pm 0.021^{3}$
\end{tabular}

Ephemeris

Orbital period P [days] $\quad 5.616531 \pm 0.000023$

Epoch $\mathrm{T}_{0}$ [BJD] $\quad 2456864.459 \pm 0.005^{4}$

Transit parameters

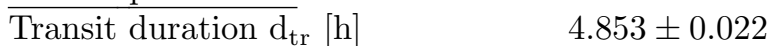

transit depth [\%] $\quad 0.934 \pm 0.024$

Spectroscopic parameters of the star

$\begin{array}{ll}\text { Effective temperature } T_{\text {eff }}[\mathrm{K}] & 6430 \pm 70 \\ \text { Surface gravity log g }[\mathrm{cgs}] & \sim 4.0 \\ \text { Spectral type } & \text { F3V-F5IV }\end{array}$

$\underline{\text { Physical parameters }}$

semi-mayor axis $[\mathrm{AU}] \quad 0.0697$

$\begin{array}{ll}\mathrm{M}_{\mathrm{v}} & 2.8 \pm 0.1^{3}\end{array}$

interstellar extinction $\mathrm{A}_{\mathrm{V}}[\mathrm{mag}] \quad 0.84 \pm 0.14$

Distance of the star d [pc] $\quad 723 \pm 30$

Star mass $\left[\mathrm{M}_{\odot}\right]$

Star radius $\left[\mathrm{R}_{\odot}\right]$

Age of the star [Gyr]

Planet mass $\mathrm{M}_{\mathrm{p}}\left[\mathrm{M}_{\mathrm{Jup}}\right]$

Planet radius $R_{\mathrm{p}}\left[\mathrm{R}_{\mathrm{Jup}}\right]$

$\lambda$ [deg] $^{6}$

$1.43 \pm 0.04^{5}$

$1.93 \pm 0.01^{5}$

$2.3 \pm 0.2^{5}$

$<1.4$

$1.87 \pm 0.03$

90

Notes. ${ }^{(1)}$ PPMXL Catalog (Roeser et al. 2010). ${ }^{(2)}$ EXODAT (Deleuil et al. 2009). (3) 2MASS (Skrutskie et al. 2006). (4) Heliocentric Julian date. ${ }^{(5)}$ Derived by using the evolutionary tracks published by Girardi et al. (2000). The values are in agreement with STAREVOL tracks. ${ }^{(6)}$ Sky-projected angle between the planetary orbital axis and the stellar rotation axis.

with PISCES at the Large Binocular Telescope (LBT). The aim of these observations was to find out whether there are any stars within a few arcsec from the target that are bright enought to be FPs (see Guenther et al. 2013 for details on this method). We detected two previously unknown stars. Star No. 1 is $11^{\prime \prime} 94 \pm 00^{\prime \prime} .02 \mathrm{~W}, 0^{\prime \prime} .30 \pm 0$ "!03 S, and has $J=15.7 \pm 0.1, K=16.2 \pm 0.1$, and star No. 2

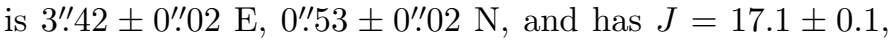
$K=16.8 \pm 0.1$ (Fig. III.7.1). Both stars are not visible in the images taken obtained with the WISE and IAC-80-cm telescopes. The colours of the two stars are not consistent with physical companions, and both are far too faint to be potential FPs. Using RV-measurements obtained with FIES@ NOT, we derived an upper limit for the mass of the companion of $\mathrm{M}_{\mathrm{p}} \leq 2.9 \mathrm{M}_{\mathrm{Jup}}$. 


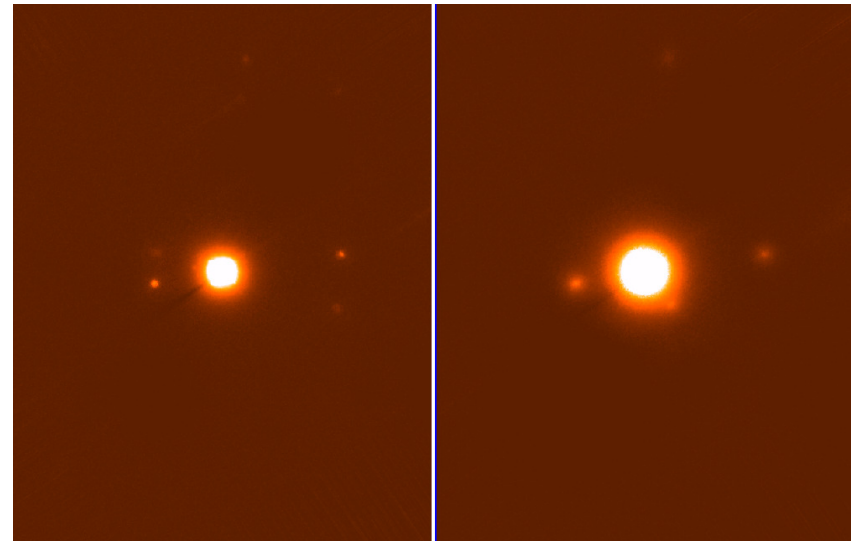

Fig. III.7.1. Images taken with the AO-system PISCES at the LBT in the $K$ (left) and in the $J$-band (right). The size of the image is only about $6 \times 8^{\prime \prime}$, north is up and east is left. Two previously unknown stars are detected at distances of $1^{\prime \prime} .96 \pm$

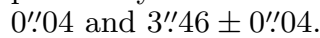

\section{Time resolved spectroscopy of LRc07_E2_0307}

Time resolved spectroscopy is a new method that allows to cofirm a planet without the need to determine its mass by means of RV-measurements. This method was pioneered by Collier Cameron et al. (2010). This method has some similarity with the classical Rossiter-McLaughlin (RM) method but has the advantage that it works also for rapidly rotating stars.

We obtained two independent data-sets. One with UVES on ESO VLT UT-2 (KUEYEN). The transit was observed on 6 August 2013. Each in-transit spectrum was exposed for $970 \mathrm{~s}$, the out of transit spectrum was exposed for $1450 \mathrm{~s}$. The in-transit observations were obtained for $2^{h} 55^{m}$, and the out-of-transit spectrum was taken $2^{h} 9^{m}$ after the end of the in-transit observations in the same night. We obtained the second data-set with the fibre-fed Echelle spectrograph FIES in the night 25-26 July 2013.

We analyzed the UVES-data in two different ways. Once by using time resolved spectroscopy as explained in Collier Cameron et al. (2010), and also by measuring the classical RM-effect. The shadow of the transiting planet is clearly seen in the time-resolved spectroscopy and the RM-analysis shows a constant shift of the RV during transit. This implies that the planet is in a polar orbit. In case of the FIES data, we only applied the classical RM-method, because of the lower S/N. This data-set confirms the UVES results (Fig. III.7.2). After having excluded all other possibilities, we therefore regard LRc07_E2_0307 as a planet. The properties of the planet are summarized in Table III.7.3.

\section{LRa02_E2_4150}

LRa02_E2_4150 (HD 295605) is a very interesting object, because it is the only candidate that is a B-star. The stellar parameters are $T_{\text {eff }}=15800 \mathrm{~K}, \log (\mathrm{g})=4.40$, which gives $M_{*} \sim 6 \mathrm{M}_{\odot}, R_{*}=3.8 \mathrm{R}_{\odot}$, which is consistent with a B5V-star. The difficulty with this object is that it

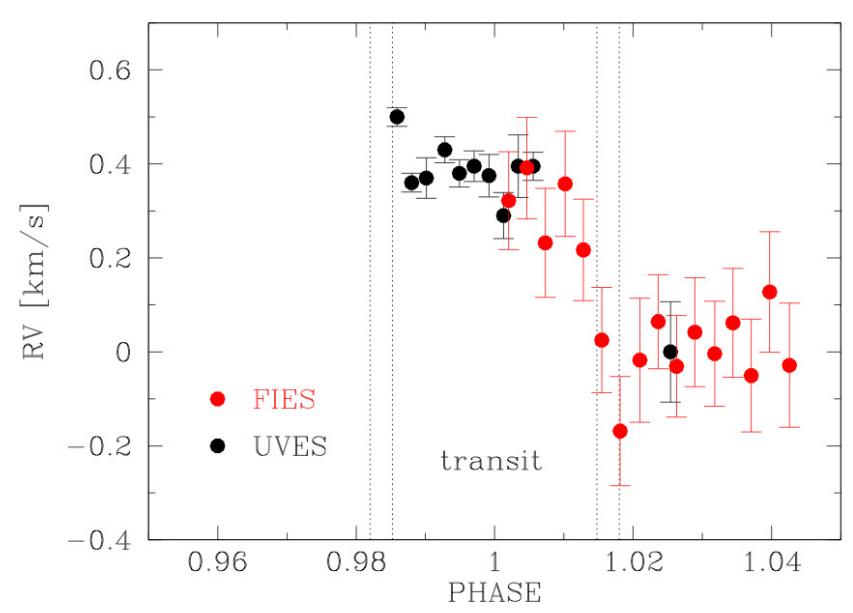

Fig. III.7.2. RV-values taken during and after the transit with UVES and FIES (instrumental offset removed). Both datasets are consistent with a planet in a polar orbit.

oscillates. On top of these oscillations there are transit-like events which have a depth of about $0.7 \%$. This would imply that the transiting object would have a size of $R_{\mathrm{p}}=$ $0.3 \mathrm{R}_{\odot}$, or $R_{\mathrm{p}}=3 \mathrm{R}_{\mathrm{Jup}}$, if there is a transiting object. Although this makes it unlikely to be a planet, or BD, we still obtained RV-measurements with CAFÉ, HARPS, the SANDIFORD, and FIES. The seeing-limited acquisition images taken with STANCAM during the RV follow-up performed with FIES, showed a sightly elongated source, suggesting that the target might be a visual binary with an angular separation of $\sim 2^{\prime \prime}$. We obtained images with the $V, R, I$, and $\mathrm{H} \alpha$ at the IAC-80-cm telescope to search for a hypothetical stellar companion or HII-region associated to the target but none was found. We thus regard the elongation of the STANCAM acquisition image as an artifact.

Unfortunately, the transit-like events have a period period of 8.17 days which is longer than the individual observing runs. We thus have to merge the RV-measurements obtained at different campaigns. Combining all the data, we derived an upper limit of about $90 \mathrm{M}_{\text {Jup }}$ for the mass of the companion. The hypothetical eclipsing object thus cannot be a stellar companion. Whether this star hosts a transiting planet, or a BD, or whether it is a FP caused by the oscillations can not be deduced from data.

\section{Discussion and conclusions}

As discussed in Sect. 2, we expected to find about 6-8 substellar-objects of A-, and B-stars. Our RV-measurements clearly demonstrate that it is possible to detect the RV-signal of a BD with an orbital period $\leq 6$ days of an A-star, despite the rapid rotation and the oscillations of these stars. Our experience with HD15082b shows that hundreds of spectra are needed to determine the mass of planet orbiting an A-star (Lehmann et al. 2014). However, the number of candidates that we found corresponds exactly to our expectation, if we assume that the frequency of massive close-in planets of A-stars is the same as that of G-stars. All our candidates are bright enough to be observed with TESS and PLATO which, 
combined with multi-colour phtometry of the transit, will eventually show whether these objects are planets, or not. The future of searching for planets of IMSs is thus bright.

Acknowledgements. We acknowledge support by the Deutsche Forschungsgemeinschaft (DFG) through grant GU464/151,GU 464/16-1, GU 464/17-1, and GU464/18-1. The groundbased observations were obtained with the VLT at the European Southern Observatory at Paranal, Chile (291.C-5028(A), 092.C0222(A), and 093.C-0027(A), with the 2.2-m and 3.5-m telescope at German-Spanish Astronomical Center at Calar Alto (H12-2.2-037, H14-2.2-043), with the Nordic Optical Telescope at La Palma (NOT runs P45-109, P45-206, P46-113, P46-215, P47-105, P47-211, P48-217, P49-205; funding from the European Union Seventh Framework Programme FP7/2013-2016 under grant agreement No. 312430, OPTICON), the 2.1-m at McDonald observatory, Texas, the 80-cm telescope of the Instituto de Astrofísica de Canarias at the observatorio del Teide, the Alfred-Jensch telescope in Tautenburg, and the Large Binocular Telescope. The NOT is operated on the island of La Palma jointly by Denmark, Finland, Iceland, Norway, and Sweden, in the Spanish Observatorio del Roque de los Muchachos of the Instituto de Astrofísica de Canarias. The 2.2-m and 3.5-m telescopes of the Centro Astronómico Hispano Alemán (CAHA) is located in the Sierra de Los Filabres (Andalucía, Southern Spain) north of Almeria. It is operated jointly by the Max-Planck-Institut for Astronomie (MPIA) in Heidelberg, Germany, and the Instituto de Astrofísica de Andalucía (CSIC) in Granada/Spain. The LBT is an international collaboration. LBT Corporation partners are: The University of Arizona on behalf of the Arizona university system; Istituto Nazionale di Astrofisica, Italy; LBT Beteiligungsgesellschaft, Germany, representing the Max-Planck Society, the Astrophysical Institute Potsdam, and Heidelberg University; The Ohio State University, and The Research Corporation, on behalf of The University of Notre Dame, University of Minnesota and University of Virginia. This publication makes use of data products from the Two Micron All Sky Survey, which is a joint project of the University of Massachusetts and the Infrared Processing and Analysis Center/California Institute of Technology, funded by the National Aeronautics and Space Administration and the National Science Foundation.

\section{References}

Albrecht, S., Winn, J. N., Johnson, J. A., et al. 2012, ApJ, 757,18

Albrecht, S., Winn, J. N., Marcy, G. W., et al. 2013, ApJ, 771,11

Alibert, Y., Mordasini, C., \& Benz, W. 2011, A\&A, 526, A63

Almenara, J. M., Deeg, H. J., Aigrain, S., et al. 2009, A\&A, 506,337

Auvergne, M., Bodin, P., Boisnard, L., et al. 2009, A\&A, 506, 411

Bear, E., \& Soker, N. 2012, ApJ, 749, L14

Beaugé, C., \& Nesvorný, D. 2012, ApJ, 751, 119

Beuermann, K., Dreizler, S., Hessman, F. V., \& Deller, J. 2012, A\&A, 543, 138

Borgniet, S., Boisse, I., Lagrange, A.-M., et al. 2014, A\&A, 561, A65

Boss, A. P. 2006, ApJ, 644, L79

Bouchy, F., Deleuil, M., Guillot, T., et al. 2011, A\&A, 525, A68

Bowler, B. P., Johnson, J. A., Marcy, G. W., et al. 2010, ApJ, 709, 396

Chaplin, W. J., Sanchis-Ojeda, R., Campante, T. L., et al. 2013, ApJ, 766, 101

Charpinet, S., Fontaine, G., Brassard, P., et al. 2011, Nature, 480, 496
Collier Cameron, A., Guenther, E., Smalley, B., et al. 2010, MNRAS, 407, 507

Deeg, H. J., Gillon, M., Shporer, A., et al. 2009, A\&A, 506, 343

Deleuil, M., Deeg, H. J., Alonso, R., et al. 2008, A\&A, 491, 889

Deleuil, M., Meunier, J. C., Moutou, C., et al. 2009, AJ, 138,649

Deleuil, M., Bonomo, A. S., Ferraz-Mello, S., et al. 2012, A\&A, 538, A145

De Marco, O., \& Soker, N. 2011, PASP, 123, 402

Galland, F., Lagrange, A.-M., Udry, S., et al. 2005, A\&A, 444, L21

Galland, F., Lagrange, A.-M., Udry, S., et al. 2006, A\&A, 447, 355

Gandolfi, D., Hébrard, G., Alonso, R., et al. 2010, A\&A, 524, A 55

Girardi, L., Bressan, A., Bertelli, G., \& Chiosi, C. 2000, A\&AS, 141, 371

Desort, M., Lagrange, A.-M., Galland, F., et al. 2009, A\&A, 506,1469

Desort, M., Lagrange, A.-M., Galland, F., et al. 2009b, A\&A, 499, 623

Guenther, E. W., Hartmann, M., Esposito, M., et al. 2009 , A\&A, 507, 1659

Guenther, E. W., Díaz, R. F., Gazzano, J.-C., et al. 2012, A\&A, 537, A136

Guenther, E. W., Fridlund, M., Alonso, R., et al. 2013, A\&A, 556, A75

Hasegawa, Y., \& Pudritz, R. E. 2013, ApJ, 778, 78

Hirano, T., Sanchis-Ojeda, R., Takeda, Y., et al. 2012, ApJ, 756,66

Huber, D., Carter, J. A., Barbieri, M., et al. 2013, Science, 342,331

Ida, S., \& Lin, D. N. C. 2005, ApJ, 626, 1045

Johnson, J. A., Howard, A. W., Bowler, B. P., et al. 2010a, PASP, 122, 701

Johnson, J. A., Bowler, B. P., Howard, A. W., et al. 2010b, ApJ, 721, L153

Jones, M. I., Jenkins, J. S., Bluhm, P., Rojo, P., \& Melo, C. H. F. 2014, A\&A, 566, A113

Kennedy, G. M., \& Kenyon, S. J. 2008, ApJ, 673, 502

Kornet, K., Różyczka, M., \& Stepinski, T. F. 2004, A\&A, 417, 151

Kunitomo, M., Ikoma, M., Sato, B., Katsuta, Y., \& Ida, S. 2011, ApJ, 737, 66

Lai, D., Foucart, F., \& Lin, D. N. C. 2011, MNRAS, 412, 2790

Laughlin, G., Bodenheimer, P., \& Adams, F. C. 2004, ApJ, 612, L73

Léger, A., Rouan, D., Schneider, J., et al. 2009, A\&A, 506, 287

Lehmann, H., Guenther, E., Sebastian, D., et al. 2015, A\&A, 578, L4

Lin, D. N. C., Bodenheimer, P., \& Richardson, D. C. 1996, Nature, 380, 606

Livio, M., \& Soker, N. 2002, ApJ, 571, L161

Mamajek, E. E. 2009, AIP Conf. Ser., 1158, 3

Massarotti, A. 2008, AJ, 135, 2287

Moutou, C., Bruntt, H., Guillot, T., et al. 2008, A\&A, 488, L47

Moutou, C., Almenara, J. M., Díaz, R. F., et al. 2014, MNRAS, 444, 2783 
Pasquini, L., Döllinger M. P., Weiss, A., et al. 2007, A\&A, Siess, L., \& Livio, M. 1999b, MNRAS, 304, 925 A\&A, 545, 473, 979 A76

Pätzold, M., Endl, M., Csizmadia, S., et al. 2012, A\&A, Silvotti, R., Schuh, S., Janulis, R., et al. 2007, Nature, 449, $545, \mathrm{~A} 6$ 189

Politano, M., Taam, R. E., van der Sluys, M., \& Willems, B. 2008, ApJ, 687, L99

Skrutskie, M. F., Cutri, R. M., Stiening, R., et al. 2006, AJ, 131,1163

Queloz, D., Bouchy, F., Moutou, et al. 2009, A\&A, 506 303

Roeser, S., Demleitner, M., \& Schilbach, E. 2010, AJ, 139, 2440

Rouan, D., Parviainen, H., Moutou, C., et al. 2012, A\&A, 537, A54

Sanchis-Ojeda, R., Fabrycky, D. C., Winn, J. N., et al. 2012, Nature, 487, 449

Schlaufman, K. C., \& Winn, J. N. 2013, ApJ, 772, 143

Sebastian, D., Guenther, E. W., Schaffenroth, V., et al. 2012, A\&A, 541, A34

Siess, L., \& Livio, M. 1999a, MNRAS, 308, 1133

Soker, N.1997, ApJS, 112, 487

Tingley, B., Endl, M., Gazzano, J.-C., et al. 2011, A\&A, 528, A97

Van Eylen, V., Lund, M. N., Silva Aguirre, V., et al. 2014, ApJ, 782, 14

Vigan, A., Patience, J., Marois, C., et al. 2012, A\&A, 544, 9

Villaver, E., \& Livio, M. 2009, ApJ, 705, L81

Williams, J. P., \& Cieza, L. A. 2011, ARA\&A, 49, 67

Winn, J. N., Fabrycky, D., Albrecht, S., \& Johnson, J. A. 2010, ApJ, 718, L145

Xue, Y., Suto, Y., Taruya, A., et al. 2014, ApJ, 784, 66

Acknowledgements: The CoRoT space mission has been developed and operated by CNES, with the contribution of Austria, Belgium, Brazil, ESA, Germany, and Spain. 